\title{
Functional assessment of the hand: reproducibility, acceptability, and utility of a new system for measuring strength
}

\author{
P HELLIWELL, A HOWE, AND V WRIGHT \\ From the Regional Rheumatology Centre, Royal Bath Hospital, Harrogate; and the Rheumatism Research \\ Unit, University Department of Medicine, General Infirmary at Leeds
}

SUMmARY A new system for measuring strength of the hand using a torsion dynamometer linked to a microprocessor is described. The system permits analysis of timed squeezes of both grip and pinch and is adjustable to all sizes of hand and degrees of hand deformity. Results obtained with the system were found to be reproducible, and the rigid device was acceptable to a group of patients with arthritic hands. In rheumatoid arthritis there is a marked reduction in maximum grip and pinch strength, together with a prolongation of the time taken to reach this maximum, and increased fatigue. The limitations of grip strength as a measure of function of the hand are discussed.

Key words: grip strength, pinch strength, rheumatoid arthritis, microprocessor.

Despite the development of indices designed to assess the function of the rheumatic hand, ${ }^{1}$ grip strength assessment remains the cornerstone of most longitudinal studies designed to show functional change in the hand. Traditionally this is measured using a Davis bag, which is essentially a pneumodynamometer. Despite the ready availability, cheapness, and portability of this apparatus as an instrument for measuring strength, the pneumodynamometer has several drawbacks. Firstly, pressure produced in the system is dependent on force multiplied by the area over which this force is applied and with the Davis bag the position of the patient's hand with regard to the bag cannot always be relied upon to be the same. Secondly, the pneumodynamometer is filled with air; since air is compressible, the system is liable to be non-linear in response ${ }^{2}$ though theoretically the magnitude of this error is likely to be small. Despite these objections results with the pneumodynamometer are acceptable and reproducible, ${ }^{3}$ though in practice the observer of three consecutive readings with this device may be unconsciously smoothing the values.

Accepted for publication 29 August 1986.

Correspondence to Dr P Helliwell, Rheumatology and Rehabilitation Research Unit, School of Medicine, 36 Clarendon Road, Leeds LS2 9PJ.
Attempts to refine the pneumodynamometer have been made. Filling the system with hydraulic fluid and enclosing the bag in an aluminium sleeve ${ }^{1}$ have been tried to overcome the aforementioned problems. Linking the pneumatic system via a transducer to a microprocessor and performing analysis of the grip time curve has also been attempted. ${ }^{4}$ These authors suggested that the rate of development of maximum grip was a more sensitive indicator of hand dysfunction than the maximum grip strength itself. Furthermore, it has been suggested that changes in the rate of development of grip serve as a better discriminator in measuring response of patients to anti-inflammatory drug therapy. ${ }^{6}$

We have used a new strain gauged torsion dynamometer linked to a microprocessor for analysis of a timed squeeze in a group of normal patients and in a group of patients with rheumatoid arthritis. We have established normal values for the grip time curve and have assessed reproducibility of successive measurements. First impressions of the new system were that the rigid device caused more discomfort to people with rheumatoid arthritis than the more malleable Davis bag. Therefore, a patient preference study was carried out in a group of patients with rheumatoid arthritis in order to assess acceptability of the new device. 


\section{Description of system}

The new digital grip analyser is shown in Fig. 1. The device consists of two cushioned aluminium bars approximately six inches long. The separation between the two handles is adjustable to suit any hand size or deformity and the design allows for measurement of finger pinch and hand grip strength. A digital readout facility in newtons force is provided with the option of holding the maximum value obtained. The grip strength analyser was interfaced with a BBC model B microcomputer. The software for use with the analyser provided a visual cue to start the test and an audible cue for the patient to release their grip.

The computer program records force in newtons every 0.2 seconds for a total time of 4.4 seconds. The grip versus time curve thus produced is graphically displayed on the monitor and can be printed out on completion of the test (Fig. 2). The curve is mathematically described by the following parameters: maximum grip strength in newtons; time to maximum value in seconds; rate of loss of grip from maximum value to the point of release of grip (described as the fatigue rate), expressed in newtons per second; an expression of the amount of fatigue in terms of the percentage loss of grip from the maximum grip strength to the point of release of the grip (this is given by the formula (MGS-FGS)100/ MGS, where MGS is maximum grip strength and FGS is final grip strength); rate of loss of grip from the point of release to baseline value (described as the release rate), expressed in newtons per second;

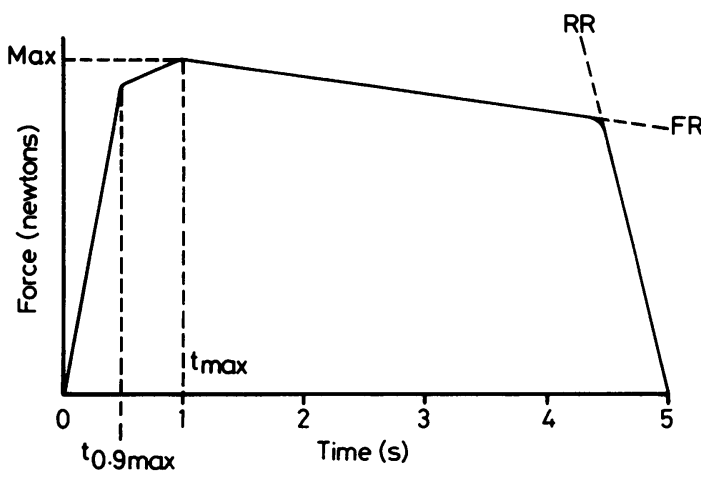

Fig. 2 Schematic representation of grip/time curve. Max $=$ maximum grip strength in newtons. $T_{\max }=$ time to maximum grip in seconds. $t_{0.9 \max }=$ time to $90 \%$ of maximum grip in seconds (see 'Discussion'). $F R=$ rate of loss of grip from maximum value to point of release in newtons per second. $R R=$ rate of loss of grip from point of release to baseline in newtons per second. Power factor is the integral of the curve in newton seconds.

the integral of the curve (described rather loosely as 'power factor'), expressed in newton seconds.

The test was found to be easy to administer and applicable to all patients with arthritic hands. Lateral pinch (between thumb pad and side of index finger) was used in all subjects. In approximately $10 \%$ of patients with rheumatoid arthritis it was necessary to adjust the handle separation. This took only a few seconds and was standardised for each

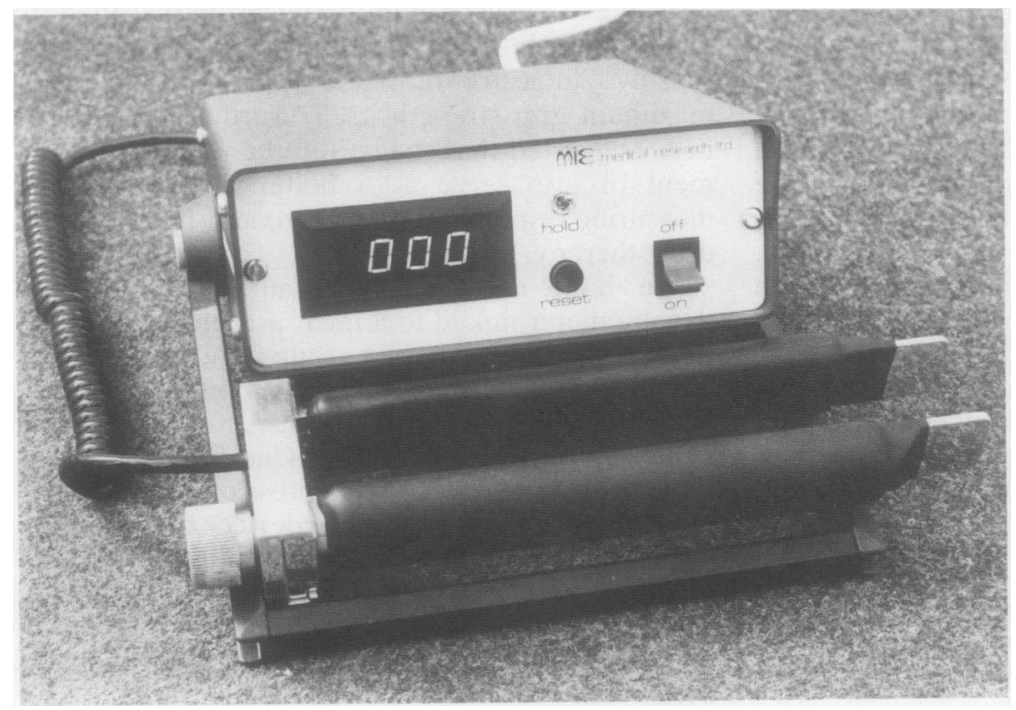

Fig. 1 Digital pinch/grip meter (MIE Medical Research Ltd). 
subject by use of a graduated scale. Narrowing the separation between the handles in some arthritic subjects does not confer on them an extra mechanical advantage: rather it enables them to employ the same techniques of grip as their counterparts who are able to grip the normally spaced handles. Provided that the amount of separation is constant, between subject comparability remains valid.

When a patient first uses the system one or two trial grips are undertaken to familiarise the patient with the machine. The patient is asked to grip as fast and as hard as possible after the signal and to hold the grip until the signal for release is given. Within seconds an analysis of the grip/time curve is presented on the monitor and if it appears satisfactory the test is completed. For a patient familiar with the system the time taken to obtain satisfactory curves for both hands, assessing grip and pinch strength, is about three minutes.

\section{Patients and methods}

In an attempt to define normality two groups of non-arthritic subjects were studied. Group A consisted of 20 members of the nursing staff of the Royal Bath Hospital, Harrogate, most of whom were women aged 18-30. Group B consisted of patients waiting to see the doctor in a general practitioner's waiting room. No patient complained of pain or stiffness in the hands. The age range of this group was 47-90 years, mean age 63, and consisted of 20 women and 10 men. Group C consisted of inpatients with rheumatoid arthritis at the Royal Bath Hospital, Harrogate. Age range for this group was from 33 to 77 years, with a mean age of 57, and consisted of 33 women and 13 men. Any differences between groups $B$ and $C$ were analysed using a $t$ test with Satterthwaite's approximation for number of degrees of freedom. ${ }^{7}$

Reproducibility of measurements was investigated by recording three successive attempts at gripping and pinching in a group of 20 subjects with rheumatoid arthritis. For the group as a whole mean values for each parameter at each attempt were calculated and the results analysed using a one way analysis of variance.

The patient preference study consisted of 26 subjects with arthritic hands (21 with rheumatoid arthritis, three with psoriatic arthritis, and two with osteoarthritis). In four subjects both hands were tested because of a difference between hands in pain experience, swelling, or deformity. Subjects were asked to squeeze each device three times, resting for a few seconds in between tests. When the Davis bag was used subjects were asked to squeeze as hard as possible for four seconds and the maximum value obtained each time was recorded. The device on which the patients were tested first was alternated to avoid any carry over effect if one device was found to be more painful to use. For each patient mean maximum grip strength with each device was recorded, together with a full Ritchie articular index ${ }^{8}$ and a modified articular index. ${ }^{9}$ The modified articular index consisted of the observer squeezing the wrist, metacarpophalangeal joints as a group, and proximal interphalangeal joints as a group and scoring as with the Ritchie articular index from 0 to 3 according to the painful response elicited. Thus the maximum score for the modified index for each hand was 9. The patients were asked at the end of

Table 1 Mean values (SEM): three consecutive grips in 20 patients with rheumatoid arthritis

\begin{tabular}{|c|c|c|c|c|}
\hline & 1 & 2 & 3 & $F$ ratio \\
\hline $\operatorname{Maximum}(\mathrm{N})$ & $91 \cdot 4(20 \cdot 4)$ & $89.5(20.0)$ & $91 \cdot 2(20 \cdot 4)$ & 0.004 \\
\hline$t_{\max }(s)$ & $2.9(0.3)$ & $2.49(0.35)$ & $2.40(0.24)$ & 1.05 \\
\hline Fatigue rate $(\mathrm{N} / \mathrm{s})$ & $13 \cdot 1(4 \cdot 6)$ & $6.75(2.5)$ & $10 \cdot 7(1.81)$ & 0.95 \\
\hline Fatigue $(\%)$ & $10 \cdot 2(1.9)$ & $11.25(1.9)$ & $11.2(1.7)$ & $0 \cdot 11$ \\
\hline Release rate $(\mathrm{N} / \mathrm{s})$ & $154.6(31)$ & $186 \cdot 3$ & 205.9 (39) & $0 \cdot 54$ \\
\hline Power factor (N.s) & $392.4(67 \cdot 5)$ & $374.7(64.2)$ & $379 \cdot 5(62 \cdot 0)$ & 0.02 \\
\hline
\end{tabular}

Table 2 Mean values (SEM): three consecutive pinches in 20 patients with rheumatoid arthritis

\begin{tabular}{|c|c|c|c|c|}
\hline & 1 & 2 & 3 & $F$ ratio \\
\hline Maximum (N) & $37.9(5 \cdot 3)$ & $36 \cdot 4(4 \cdot 9)$ & $39 \cdot 1(5 \cdot 7)$ & 0.06 \\
\hline$t_{\max }(s)$ & $2.36(0 \cdot 3)$ & $1.76(0.3)$ & $2 \cdot 06(0 \cdot 3)$ & 0.98 \\
\hline Fatigue rate $(\mathrm{N} / \mathrm{s})$ & $6.65(2.6)$ & $3 \cdot 2(0 \cdot 6)$ & $7 \cdot 1(2 \cdot 0)$ & 1.23 \\
\hline Fatigue $(\%)$ & $15 \cdot 1(3 \cdot 0)$ & $14 \cdot 2(2 \cdot 8)$ & $18 \cdot 5^{\circ}(3 \cdot 8)$ & $0 \cdot 5$ \\
\hline Release rate $(\mathrm{N} / \mathrm{s})$ & $94.7(14.0)$ & $94 \cdot 1(13 \cdot 3)$ & $100 \cdot 5(16 \cdot 0)$ & 0.06 \\
\hline Power factor (N.s) & $155 \cdot 2(22.9)$ & $148 \cdot 2(20 \cdot 6)$ & $154 \cdot 1(23 \cdot 5)$ & 0.03 \\
\hline
\end{tabular}


the test the following question: "If you had to squeeze these devices each day as part of your assessment, which would you prefer?' Device preference was thus noted. Any difference between the two groups was analysed using the Fisher exact probability test.

\section{Results}

RE P R O D U C I B I L IT Y

Reproducibility for grip and pinch is shown in Tables 1 and 2 respectively. For the group as a whole, reproducibility was acceptable for all parameters for both grip and pinch. On an individual basis the maximum grip strength and power factor were highly reproducible, but the values for $t_{\max }$ tended to be somewhat erratic. Therefore caution needs to be taken when studying individuals or small groups.

COMPARATIVE STUDIES

Mean values for the three groups for grip and pinch strength are presented in Tables 3 and 4 respectively.

Little difference was seen between the two nonarthritic groups despite the marked difference in age range. Values for maximum grip strength showed that in rheumatoid arthritis grip strength was approximately $25 \%$ that of a non-arthritic population, with a reduction in pinch strength of approximately $40 \%$. Occasionally pinch strength exceeded grip strength in patients with rheumatoid arthritis, and many patients commented that they relied on tripod or lateral pinch in their everyday activities. Time to maximum grip strength in the rheumatoid group was significantly prolonged for grip, while pinch times were also prolonged but not significantly more than controls. Compared with the elderly normal population, subjects with rheumatoid arthritis showed

Table 3 Mean values (SEM) for grip strength of nurses $(A)$, non-rheumatic patients $(B)$, and patients with rheumatoid arthritis $(C)$

\begin{tabular}{|c|c|c|c|c|}
\hline & \multicolumn{3}{|l|}{ Group } & \multirow{2}{*}{$\begin{array}{l}p \text { Value } \\
(B \vee C)\end{array}$} \\
\hline & $A$ & $B$ & $C$ & \\
\hline $\operatorname{Maximum}(\mathrm{N})$ & $256(16)$ & $238(16)$ & $60(5)$ & $<0.001$ \\
\hline$t_{\operatorname{mix}}(s)$ & $1 \cdot 5(0 \cdot 2)$ & $1 \cdot 6(0 \cdot 1)$ & $2 \cdot 3(0 \cdot 2)$ & $<0 \cdot 005$ \\
\hline $\begin{array}{l}\text { Fatigue ratc } \\
(\mathrm{N} / \mathrm{s})\end{array}$ & $23(4 \cdot 0)$ & $12 \cdot 3(1.2)$ & $6 \cdot 8(2 \cdot 5)$ & $<0 \cdot 1$ \\
\hline Fatigue $(\%)$ & $11 \cdot 2(1 \cdot 4)$ & $7 \cdot 1(0 \cdot 9)$ & $11 \cdot 8(1.7)$ & $<0.025$ \\
\hline $\begin{array}{l}\text { Release rate } \\
(\mathrm{N} / \mathrm{s})\end{array}$ & $584(65)$ & $432(45)$ & $125(13)$ & $<0.001$ \\
\hline $\begin{array}{l}\text { Power factor } \\
\text { (N.s) }\end{array}$ & $1107(71)$ & $1133(74)$ & $255(29)$ & $<0.001$ \\
\hline
\end{tabular}

Table 4 Mean values (SEM) for pinch strength of nurses $(A)$, non-rheumatic patients $(B)$, and patients with rheumatoid arthritis $(C)$

\begin{tabular}{|c|c|c|c|c|}
\hline & \multicolumn{3}{|l|}{ Group } & \multirow{2}{*}{$\begin{array}{l}p \text { Value } \\
(B \vee C)\end{array}$} \\
\hline & $A$ & $B$ & $C$ & \\
\hline Maximum (N) & $83(3)$ & $78(5)$ & $32(2)$ & $<0.001$ \\
\hline $\begin{array}{l}t_{\max }(s) \\
\text { Fatigue rate }\end{array}$ & $1.5(0.3)$ & $1.6(0.2)$ & $2 \cdot 0(0 \cdot 2)$ & $<0 \cdot 2$ \\
\hline$(\mathrm{N} / \mathrm{s})$ & $7 \cdot 8(1 \cdot 1)$ & $6.5(0.8)$ & $3.2(0.6)$ & $<0.005$ \\
\hline Fatigue (\%) & $13(2 \cdot 2)$ & $10 \cdot 5(1 \cdot 3)$ & $14.8(2.3)$ & $<0 \cdot 1$ \\
\hline $\begin{array}{l}\text { Release rate } \\
(\mathrm{N} / \mathrm{s})\end{array}$ & $195(24)$ & $173(20)$ & $83(7)$ & $<0.001$ \\
\hline $\begin{array}{l}\text { Power factor } \\
\text { (N.s) }\end{array}$ & $362(15)$ & 354 (23) & $139(12)$ & $<0.001$ \\
\hline
\end{tabular}

considerably more fatigue during the grip test, and the trend was towards increased fatigue in the pinch test, though this did not achieve statistical significance.

\section{INTER-RELATIONSHIP OF STRENGTH}

VA R I A B L ES

Table 5 gives the Pearson's $r$ correlation coefficients for maximum grip strength compared with the additional parameters for the three groups of patients studied. As expected, maximum grip strength was strongly related to power factor. Maximum grip strength was also strongly related to release rate in these three groups, though this may not be so for other disease states such as, for example, myotonia. There was no correlation between maximum grip strength and time taken to reach this maximum value, so these two variables provide independent information on the grip. Maximum grip strength was positively related to fatigue rate, as expected. The maximum grip strength was independent of the percentage fatigue, though there was a trend towards a negative relationship in the groups studied.

Table 5 Correlations between variables in the three groups

\begin{tabular}{llcccc}
\hline & & Grip RA & Pinch RA & $\begin{array}{l}\text { Grip } \\
\text { group A }\end{array}$ & $\begin{array}{l}\text { Grip } \\
\text { group B }\end{array}$ \\
\hline $\operatorname{Max} v \mathrm{t}_{\text {max }}$ & $-0 \cdot 09$ & $0 \cdot 13$ & $-0 \cdot 13$ & $-0 \cdot 1$ \\
$\operatorname{Max} v$ FR & $0 \cdot 47^{*}$ & $0 \cdot 51^{*}$ & $0 \cdot 24$ & $0 \cdot 45^{*}$ \\
$\operatorname{Max} v$ F\% & $-0 \cdot 29^{*}$ & $-0 \cdot 20$ & $-0 \cdot 16$ & $-0 \cdot 25$ \\
$\operatorname{Max} v$ RR & $0 \cdot 87^{*}$ & $0 \cdot 78^{*}$ & $0 \cdot 61^{*}$ & $0 \cdot 81^{*}$ \\
$\operatorname{Max} v$ PF & $0.99^{*}$ & $0.99^{*}$ & $0 \cdot 98^{*}$ & $0 \cdot 99^{*}$ \\
\hline
\end{tabular}

${ }^{*}$ Denotes significant at $5 \%$ level.

$\mathrm{RA}=$ rheumatoid arthritis; $\mathrm{A}=$ nurses; $\mathrm{B}=$ non-rheumatic patients; $\mathrm{FR}=$ fatigue ratio; $\mathrm{F}=$ fatigue; $\mathrm{RR}=$ release rate $\mathrm{PF}=$ power factor. 
Table 6 Pneumodynamometer and torsion dynamometer: patient preference study in 26 patients with rheumatoid arthritis* $^{*}$

\begin{tabular}{lccll}
\hline & PD preference & TD preference & DK & p Value \\
\hline Number & 16 & 13 & 1 & \\
Sex & $4 \mathrm{M}, 12 \mathrm{~F}$ & $3 \mathrm{M}, 10 \mathrm{~F}$ & \\
MAI & $3 \cdot 71(0 \cdot 54)$ & $3 \cdot 58(0 \cdot 62)$ & NS \\
RI & $18 \cdot 2(2 \cdot 07)$ & $15 \cdot 5(1 \cdot 85)$ & NS \\
MGS (N) & $273(44 \cdot 3)$ & $301 \cdot 5(62 \cdot 9)$ & NS \\
MGS (mmHg) & $92.4(8 \cdot 7)$ & $93 \cdot 2(13 \cdot 5)$ & \\
\hline
\end{tabular}

$\mathrm{PD}=$ pneumodynamometer; $\mathrm{TD}=$ torsion dynamometer; $\mathrm{DK}=$ don't know; MAI =modified articular index; $\mathrm{RI}=$ Ritchie articular index; MGS=maximum grip strength.

Values are means (SEM).

${ }^{*}$ In four patients both hands were tested because of a difference between hands in pain experience.

\section{PATIENT PREFERENCE STUDY}

Results of the patient preference study are presented in Table 6 . Sixteen patients preferred the pneumodynamometer rather than the torsion dynamometer and 13 the reverse for the hand tested, with one patient undecided. There was no particular preference by gender and the values for the modified articular index and the Ritchie articular index were not significantly different for both groups.

\section{Discussion}

Grip strength assessment is frequently used in clinical trials and has been shown to be a sensitive indicator of disease activity. ${ }^{9}$ Grip strength is a composite measure and may be influenced by dysfunction in muscles, tendons, and any of the small joints of the hand and wrist. In addition, accurate reliable assessment requires maximal effort on every occasion. The patients may be motivated for a variety of reasons and this motivation may be influenced in many ways, consciously or unconsciously, by the assessor. Thus although reproducibility figures outside the trial may be acceptable, other factors may be contributing to a change in grip strength in a longitudinal trial design. With these limitations in mind a more detailed assessment of hand function has been designed. ' Although these functional indices are undoubtedly more comprehensive and provide a better guide to overall hand function, the time required to complete each index is prohibitive for clinical trial work, where many patients may be assessed in a session.

As a measure of grip and pinch strength we feel the system described here has several advantages compared with the Davis bag. The rigid strain gauged device provides more accurate data for immediate display. The design of the handles is such that the instrument is not sensitive to changes of position of the applied force, which is a problem with the pneumodynamometer. The main advantage of the digital analyser, however, is that a more in depth analysis of a timed grip can be obtained by interfacing with a microcomputer. As we have shown this provides information on the dynamic quality of grip in addition to the maximum grip strength. Furthermore, software is available to permit analysis of grip strength endurance, which may be of value in certain circumstances, such as stroke rehabilitation. This facility may also be used for assessing treatment efficacy in, for example, myasthenia gravis.

First impressions were that the rigid device was more uncomfortable for the patient to use than the softer Davis bag, but the patient preference study showed that this was not so in a group of patients with arthritic hands. The results show that preference is independent of the degree of pain in the joints of the hand and wrist, and our overall impression is that if one device caused pain on squeezing then the other device also caused pain. Patients chose a particular device on the basis of how well they thought that device represented their grip strength. In other words, if they found the Davis bag slipping out of their hand then they would choose the rigid device. Patients choosing the Davis bag found the rigid device somewhat unwieldly despite its adjustable handles for any hand size or deformity.

No attempt was made to give the patients a choice between peak or sustained grip during the preference study. It has been shown by Grindulus and Calverley that patients with rheumatoid arthritis prefer peak grip assessment. ${ }^{10}$ These workers found that very few of their patients could sustain a grip for five seconds. We found initially that having an eight second timed grip was too long for arthritic patients, but once the program was changed to a 4.4 second timed grip all patients were able to grip adequately for this period.

Reproducibility of maximum grip strength and power factor using this system compares favourably with reported reproducibility of the pneumodynamometer. ${ }^{3}$ Reproducibility of $t_{\max }$ and fatigue rate on an individual basis is not very good and caution must be used in interpreting these parameters on individual patients. For a group of patients as a whole reproducibility is adequate. In the normal groups and in the patients we studied the area under the curve, fatigue rate, and release rate were closely related to maximum grip strength and probably do not provide any additional information. The values $t_{\max }$ and percentage fatigue were inde- 
pendent of maximum grip strength, and in this group of patients these three values are of the greatest interest.

Patients with rheumatoid arthritis had approximately a $75 \%$ reduction in maximum grip strength and a $60 \%$ reduction in maximum pinch strength compared with non-arthritic controls. These figures compare favourably with those obtained by other workers. ${ }^{1112}$ Quite often lateral pinch is stronger than grip strength in rheumatoid arthritis, and patients frequently rely on this manoeuvre in their everyday activities. In addition, surgical stabilisation of the first metacarpophalangeal joint is likely to augment the execution of this movement, and we are currently studying the effect of surgical procedures on the function of the hand in this way.

Patients with rheumatoid arthritis take a significantly longer time to reach maximum grip strength than normal subjects. This has been noted before by Myers et al, ${ }^{4}$ though the absolute values obtained were quite different from ours. There is invariably an initial steep rise in the grip time curve with a subsequent slower climb to peak grip. If the time to $90 \%$ maximum is estimated this second slow climb can be eliminated and this possibly explains the difference between our results and those obtained by the New Zealand group. We have now changed the program to measure time to $90 \%$ of maximum grip and have obtained times equivalent to those of the New Zealand group.

It has been suggested that prolongation of time to maximum grip is equivalent to the subjective experience of stiffness in rheumatoid disease. ${ }^{13}$ This may result from several factors. Firstly, increased resistance to passive motion in the metacarpophalangeal joint has been recorded by Wright and Johns, ${ }^{14}$ though more recent studies with a new finger arthrograph moving the joint laterally have been unable to confirm these findings. ${ }^{15}$ Secondly, pain in the small joints of the hand and wrist may be inhibiting the rate of development of the grip. Thirdly, inherent disease in the muscles may be contributing. Electrophysiological techniques have shown that both subclinical polymyositis and abnormal muscular fatiguability are common in rheumatoid arthritis. ${ }^{16} 17$

Ultimately, the utility of the information on the dynamic qualities of grip stength would be determined in therapeutic intervention studies. Myers $e t$ al have suggested that the rate of development of grip is a better discriminator than maximum grip $\frac{\square}{\infty}$ strength for measuring response to anti-inflammatory drugs. ${ }^{6}$ We are currently studying the $\stackrel{\vec{\rho}}{\rightarrow}$ diurnal variation of these parameters in untreated 0 patients with rheumatoid arthritis, in addition to the $\overline{0}$ effect of standard therapies such as anti-inflam- $\frac{\bar{c}}{2}$ matory drugs, physiotherapy, intra-articular steroid, and pulsed intravenous steroid.

This work was supported by research grants from the Arthritis and Rheumatism Council. We thank Miss Anne Laville and Mrs Sheila Coombes for help in preparation of the manuscript.

\section{References}

1 Clawson D K, Souter W A, Carthum C J, et al. Functional $\omega$ assessment of the rheumatoid hand. Clin Orthop 1977: 77: N 203-10.

2 Kondraske G V. Measurement of the quality of hand contractions. Med Biol Eng Comput 1985; 23: 399.

3 Lee P, Baxter A, Dick W C. Webb J. An assessment of grip strength measurement in rheumatoid arthritis. Scand J Rheumatol 1974; 3: 17-23.

4 Myers D B, Grennan D M, Palmer D G. Hand grip function in paticnts with rhcumatoid arthritis. Arch Phys Med Rehabil 1980; 61: 369-73.

5 Duym B W, Pfurtscheller G. Measurement of the quality of .V hand contractions. Med Biol Eng Comput 1984; 22: 245-50.

6 Myers D B, Wilson K, Palmer D G. Relative changes in maximum grip strength, work and power output during hand grip assessment of rheumatoid patients in a drug trial. Proc Univ Otago Med Sch 1979; 57: 64-5.

7 Snedecor G W, Cochran W G. Statistical methods. 7th ed. Iowa: Iowa State University Press, 1980: 7.

8 Ritchie D M, Boyle J A, McInnes J M, et al. Clinical studies $\overline{\vec{O}}$ with an articular index for the assessment of joint tenderness in patients with rheumatoid arthritis. $Q J$ Med 1968; 37: 393-406.

9 Rhind V M, Bird H A, Wright V. A comparison of clinical assessments of disease activity in rhcumatoid arthritis. Ann Rheum Dis 1980; 39: 135-7.

10 Grindulus K A, Calverley M. Grip strength: peak or sustained pressure in rheumatoid arthritis? J Chron Dis 1983; 36: 855-8.

11 Jones A R, Unsworth A, Haslock I. A microcomputer $\exists$ controlled hand assessment system used for clinical measurement. Eng Med 1985; 14: 191-8.

12 Walker P S, Davidson M D, Erkman M J. An apparatus to assess function of the hand. J Hand Surg 1978; 3: 189-93.

13 Myers D, Wilson K, Palmer D G. An objective measurement of change in morning stiffness. Rheumatol Int 1981; 1: 135-7.

14 Wright V, Johns R J. Quantitative and qualitative analysis of joint stiffness in normal subjects and in patients with connective $\mathrm{N}$ tissue discase. Ann Rheum Dis 1961; 20: 36-46.

15 Howe A. Microprocessor mediated metacarpophalangeal joint $\mathrm{N}$ stiffness measurement. University of Leeds, 1985. (PhD thesis.)

16 Steinberg V L, Wynn Parry C B. Electromyographic changes in $\mathrm{C}$ rheumatoid arthritis. $\mathrm{Br}$ Med J 1961; i: 630-2.

17 Lenman J A R. Potter J L. Electromyographic measurement of fatigue in rheumatoid arthritis and neuromuscular disease. $A n n$ Rheum Dis 1966: 25: 76-84. 